\title{
Thermography for the Diagnosis of Acute Inflammation in the Paranasal Sinus
}

\author{
Tadashi Ishimaru $^{1}$ Hitomi Ishimaru ${ }^{1}$ \\ ${ }^{1}$ Department of Otorhinolaryngology, Hyotan-machi ENT Clinic, \\ Kanazawa, Ishikawa, Japan \\ Int Arch Otorhinolaryngol 2020;24:e215-e220.
}

\author{
Address for correspondence Tadashi Ishimaru, MD, PhD, Department \\ of Otorhinolaryngology, Hyotan-machi ENT Clinic, Hyotan-machi \\ 2-13, Kanazawa, Ishikawa 9200845, Japan \\ (e-mail: taishimaru-alg@umin.ac.jp).
}

\begin{abstract}
Keywords

- Thermography

- sinusitis

- paranasal sinuses

- maxillary sinus

- inflammation
\end{abstract}

\section{Introduction}

Sinusitis is a commonly encountered disease in the outpatient clinic. Inflammation of the paranasal sinus is primarily diagnosed via X-ray imaging, although the precise diagnosis of sinusitis depends upon computed tomography (CT) scanning. If an imaging study that does not involve X-rays could be conducted for the diagnosis of sinusitis, it would be more useful and safer for patients.

The applications of thermography in otorhinolaryngology have been reported by pioneer studies. ${ }^{1-3}$ Sergeev et $\mathrm{al}^{3}$ examined the difference between temperatures measured by a thermometer in contact with the inferior turbinate and those measured by infrared thermography of the face and found a relationship between the temperatures of the inferior turbinate and facial skin. Although this work indicated the usefulness of thermography, the technique did not yield visual results.

Because tissue temperature increases during inflammation, heat distribution on the face is possibly influenced by sinusitis. Previous researchers who used thermography reported that facial temperatures near the paranasal sinus increased when sinusitis was observed. ${ }^{3-5}$ Because the increase in the temperature differed between the two sides, we received

September 23, 2018

accepted

September 8, 2019
DOI https://doi.org/

10.1055/s-0039-1698778. ISSN 1809-9777.
Copyright (c) 2020 by Thieme Revinter

Publicações Ltda, Rio de Janeiro, Brazil
License terms

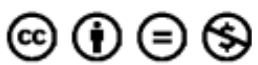


could effectively differentiate between the right and left sides through observation. ${ }^{4}$

Facial temperatures are easily influenced by individual differences and the environment. Haddad et $\mathrm{al}^{6}$ reported the heat distribution on the face in a healthy subject in a heatcontrolled room. The preparations for the thermographic examination included the regulation of room temperature, humidity, airflow, window size, electric lights etc. The effects of the environment on the examination room are reduced by digital technology; therefore, thermography may be a useful device in clinical settings.

Recently, the development of a thermographic camera for the iPhone (Apple Inc., Cupertino, CA, US) or iPad (Apple Inc.) using the iOS (Apple Inc.) system has made thermography easy to use. We examined both thermography and postdigital image processing to analyze differences between subjects with and without sinusitis.

\section{Materials and Methods}

\section{Subjects}

The present study included 10 control subjects ( 5 males and 5 females, aged [mean \pm standard deviation] $34 \pm 15$ years) and 20 patients with sinusitus ( 9 males and 11 females, aged $43 \pm 14$ years). The subjects who were declared disease-free and were diagnosed as free from nasal disease by anterior rhinoscopy were included on the study as controls. Of the 20 patients with acute rhinosinusitis (ARS), 16 were chosen according to the guidelines of the European Rhinology Society. ${ }^{7}$ Two patients with a postoperative maxillary cyst (PMC), 1 patient with acute exacerbation of chronic sinusitis (AECS), and 1 patient with odontogenic maxillary sinusitis (OMS) were included among the patients with sinusitis. The diagnoses of PMC, AECS, and OMS were made based on acute inflammation symptoms, past history, nasal cavity observations, X-ray imaging, and CT scans, and these conditions were included in the definition of "sinusitis." All patients had complaints of maxillary pain, headache, and/or rhinorrhea.

According to the laterality of the shadow of the maxillary sinus on X-ray imaging, the patients were divided into right, left, and bilateral groups. Patients with frontal sinusitis and no maxillary sinusitis were included into the "others" group (-Table 1). All subjects provided informed written consent. Each subject received a small monetary award in the form of a gift card. The experimental protocol was approved by the Review Board of the Ishikawa Medical Association in Japan.

\section{Methods}

A FLIR-ONE (FLIR Inc., Wilsonville, OR, US) thermographic camera, which has automatic calibration, with an iPod-nano (Apple Inc.) were used, and all thermographic images were taken at room temperature. Thermographic image files (with $240 \times 320$ pixels of resolution and $0.1^{\circ}$ Cof thermal sensitivity) were loaded to a personal computer by FLIR TOOLS (FLIR Inc.). These files were processed using the ImageJ (National Institutes of Health, Bethesda, MD, US) and LabVIEW8.1
Table 1 Characteristics of the patients with sinusitis

\begin{tabular}{|c|c|c|c|c|}
\hline Side & Gender & Age & Diagnosis & $\begin{array}{c}\text { Body } \\
\text { temperature }\end{array}$ \\
\hline \multirow[t]{6}{*}{ Right } & Female & 19 & ARS & 36.2 \\
\hline & Male & 31 & ARS & 36.4 \\
\hline & Male & 32 & OMS & 36.4 \\
\hline & Male & 36 & ARS & 36.7 \\
\hline & Female & 54 & AECS & 36.9 \\
\hline & Male & 66 & ARS & 36.3 \\
\hline \multirow[t]{6}{*}{ Left } & Female & 23 & ARS & 36.7 \\
\hline & Female & 39 & ARS & 36.9 \\
\hline & Female & 52 & PMC & 36.6 \\
\hline & Female* & 53 & ARS & 36.6 \\
\hline & Female & 64 & PMC & 37.0 \\
\hline & Male & 69 & ARS & 36.4 \\
\hline \multirow[t]{6}{*}{ Bilateral } & Male & 39 & ARS & 36.5 \\
\hline & Male & 41 & ARS & 36.4 \\
\hline & Male & 44 & ARS & 36.3 \\
\hline & Male & 45 & ARS & 36.8 \\
\hline & Female & 46 & ARS & 36.9 \\
\hline & Female & 50 & ARS & 36.7 \\
\hline \multirow[t]{2}{*}{ Others } & Female & 29 & ARS & 35.8 \\
\hline & Female & 29 & ARS & 37.7 \\
\hline
\end{tabular}

Abbreviations: ARS, acute rhinosinusitis; PMC, postoperative maxillary cyst; AECS acute exacerbation of chronic sinusitis; OMS odontogenic maxillary sinusitis.

Notes: "Others" indicate the subjects with frontal sinusitis without maxillary sinusitis. ${ }^{*}$ The subject in - Fig. 2.

(National Instruments, Austin, TX, US) software. The indications of spot temperatures were superimposed in the upper region of the original thermograph by the FLIR-ONE, such as "Spot1 (Sp1)." The maxillary partial thermographic image was cropped from the original thermographic image and resized to $200 \times 100$ pixels using the ImageJ. The borderlines of the maxillary part of the thermographic image included the lower eyelids, nostrils, and right and left lateral terminals ( - Fig. 1). The converted thermographic image was labeled as the standard-sized thermographic (SST) image.

The average SST image was created by taking the average of every pixel in the SST images of the subjects. The relative thermographic image was created on the basis of the difference between the original pixels and the average of all pixels of the thermography. This is how the SST image was converted to a relative SST image.

The axillary body temperature was measured using a contact thermometer.

The differences between every pixel in the image were statistically analyzed using the $t$ test. The statistical analysis of the images was performed inside of the face outline without background using the LabVIEW8.1 software. The Pearson correlation and the paired $t$ test were performed 
using the Statistical Package for the Social Sciences (SPSS, SPSS Inc., Chicago, IL, US) software, version 11.0.

The average X-ray imaging of the maxillary sinusitis on the right, left, and both sides was created using the ImageJ.

\section{Results}

\section{$X$-ray Imaging in Patients with Sinusitis}

$\mathrm{X}$-ray imaging was performed in the patients with sinusitis (using the Waters method). The shadow of the maxillary sinus was observed in 18 patients, and the shadow of the frontal sinus without maxillary sinusitis was observed in 2 patients. The patients in whom the shadow of the maxillary sinus was observed were categorized into 3 groups: right ( $n=6 ; 3$ males and 3 females), left $(n=6 ; 1$ male and 5 females), and bilateral ( $n=6 ; 4$ males and 2 females).

\section{Heat Distribution on the Faces of Individuals with and without Sinusitis}

The typical heat distribution on the face of a 60 -year-old female without sinusitis is shown in - Fig. 1. The shape of the paranasal sinus was not observed.

After setting the threshold (Th) level, a unique pattern of heat distribution was observed in the patients with sinusitis. The reference temperature point, Sp1 (-Fig. 2A), was located on the right terminal of the maxillary region. The Th was experimentally calculated using the following formula: Th $=\mathrm{Sp} 1+1.4\left({ }^{\circ} \mathrm{C}\right)$. The pattern of heat distribution (hereafter referred to as "unique pattern") was similar to the shape of the paranasal sinus ( $\mathbf{F i g}$. 2A). The X-ray image was also presented (-Fig. 2B). This unique pattern was observed in 13 patients, whereas no pattern was observed in 1 patient. Whole face patterns were observed in 6 patients. This unique pattern was not observed in the subjects without sinusitis when the Th was set according to the formula of - Fig. 2A. A typical case without sinusitis is presented in - Fig. 3; the same subject as in -Fig. 1.

\section{Relationship between Body Temperature and Average Facial Temperatures}

The relationship between axillary body temperature by thermometer (ABTT) and average facial temperatures by thermography (AFTT) was studied in all of the subjects. The ABTT and AFTT (mean \pm standard deviation [SD]) for the control subjects were of $36.6 \pm 0.3^{\circ} \mathrm{C}$ and $38.8 \pm 1.5^{\circ} \mathrm{C}$ respectively, and for the patients with sinusitis, those values were of $37.7 \pm 0.4^{\circ} \mathrm{C}$ and $40.4 \pm 1.0^{\circ} \mathrm{C}$ respectively. The differences between ABTT and AFTT were significant in the control subjects (paired $t$ test, $p<0.005$ ) and in the patients with sinusitis $(p<0.005)$. The correlations between ABTT and AFTT in the sample were also studied, but they were not significant (control subjects: Pearson, $\mathrm{r}=-0.399, p=0.254$, $n=10$; patients with sinusitis: Pearson, $\mathrm{r}=0.309, p=0.185$, $n=20$ ).

\section{Average Heat Distribution in the Maxillary Region}

The heat distribution in average SST images $(n=10)$ indicated that the nose had the lowest temperature in the face

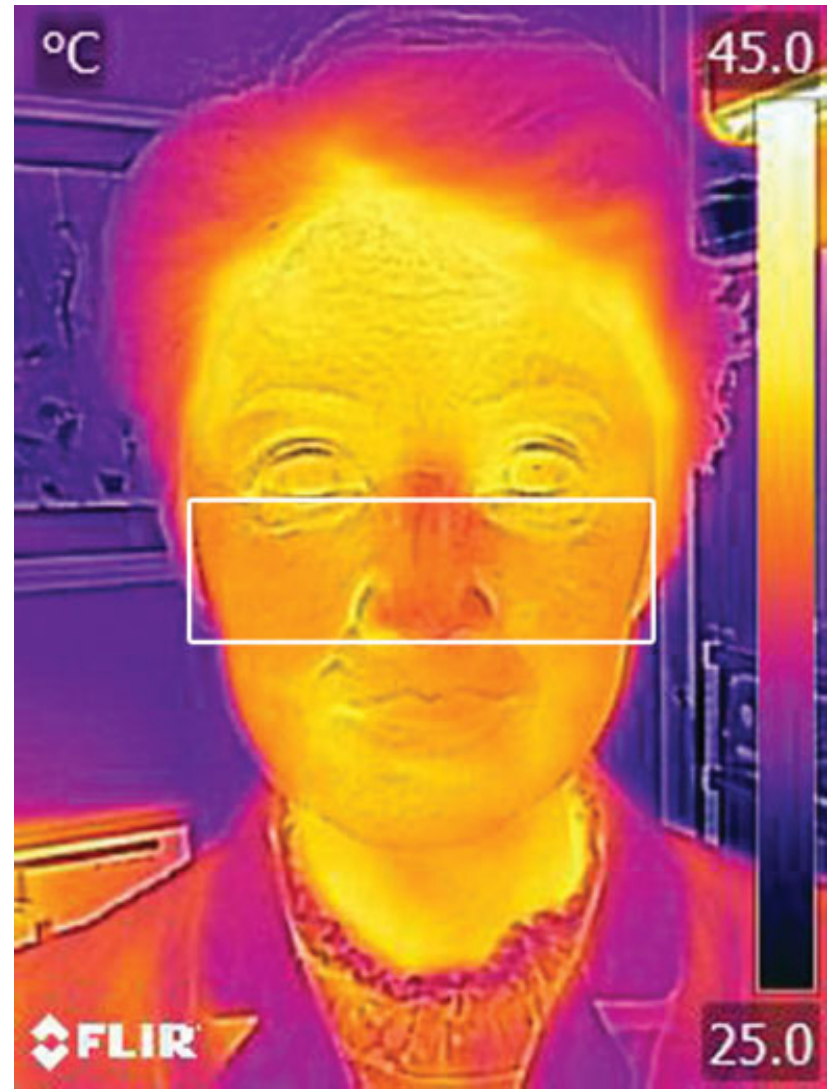

Fig. 1 Typical heat distribution. The thermography image of a 60year-old subject without sinusitis is shown. The temperature $\left({ }^{\circ} \mathrm{C}\right)$ range is indicated as a bar. The borderline of the maxillary part is indicated as a white line.

( Fig. 4A). The borderline between the face and the background was unclear in the average image ( - Fig. 4A-D). The horizontal heat distribution in SST images indicated that the center, the location of the nose, had the lowest temperature in the face (-Fig. 5). When sinusitis was present, a region with high temperatures expanded to both sides ( - Fig. 4B, C, D). The difference in laterality of the sinusitis was unclear ( -Figs. 4B, C, D). Although these unclear tendencies were also observed in the average relative SST images, the difference in heat distribution patterns between the patients with sinusitis and the control subjects was more pronounced (-Figs. 4E, F, G, H).

\section{Difference in Heat Distribution on the Faces of the study groups}

The difference between the SST images of the patients with maxillary sinusitis $(n=18)$ and of those of the control subjects $(n=10)$ was statistically analyzed. The temperatures of the whole face were significantly higher in the patients with sinusitis than in the control subjects ( $t$ test, $p<0.05$ ). The unique pattern observed in the paranasal sinus shape was not visualized (data not shown).

The difference between the relative SST images of the patients with sinusitis and of those of the control subjects was also statistically analyzed. Statistically significant increases in temperature occurred when maxillary sinusitis 


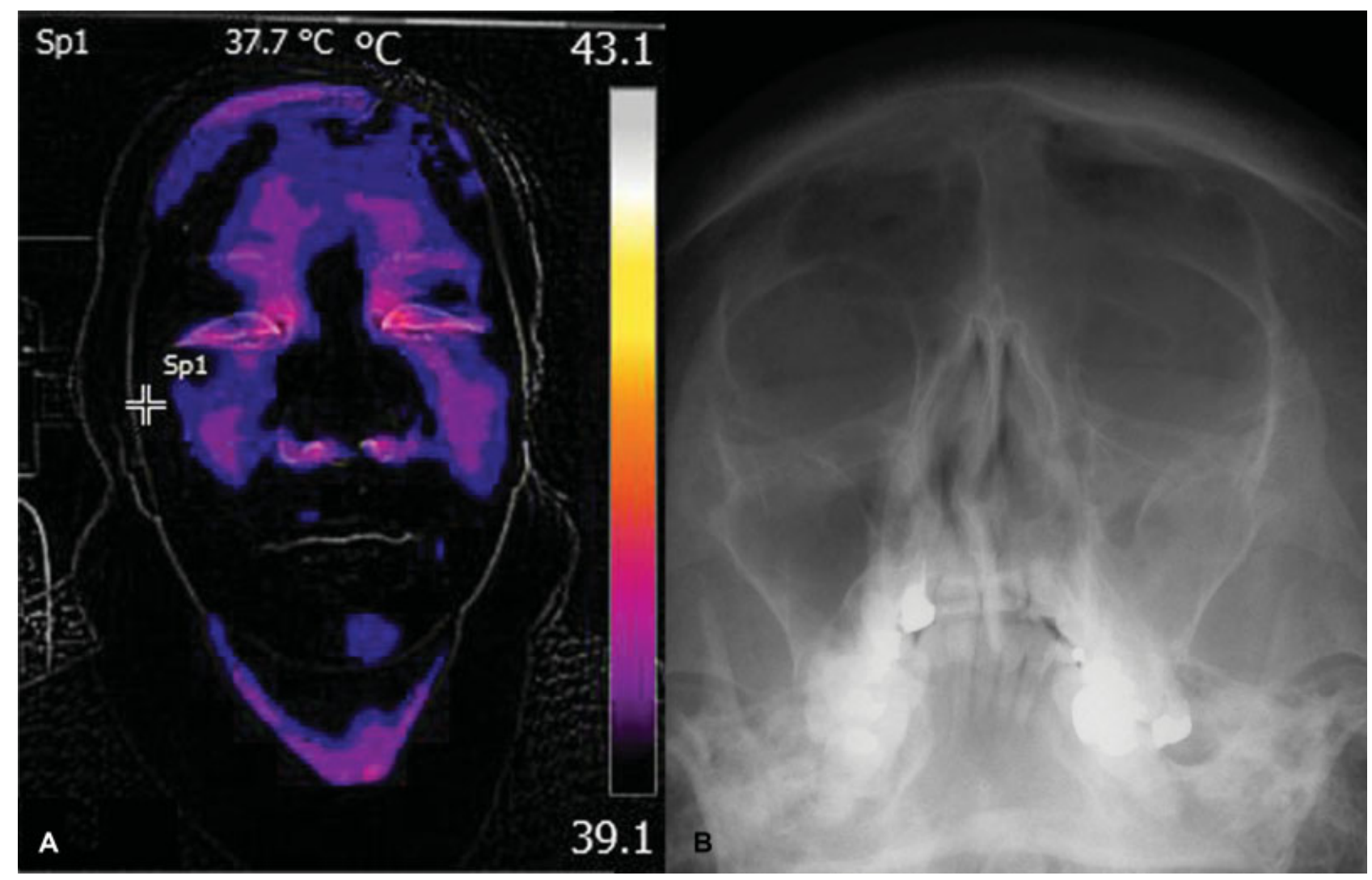

Fig. 2 Heat distribution and X-ray imaging of a patient with sinusitis. The threshold level of temperature is set at $39.1^{\circ} \mathrm{C}$. The pattern of heat distribution is similar to the shape of the paranasal sinus $(\mathrm{A})$. The temperature $\left({ }^{\circ} \mathrm{C}\right)$ range is indicated as a bar. X-ray imaging of the same patient (B). The subject is a 53-year-old female with acute sinusitis (-Table 1*).

was observed ( $t$ test, $p<0.05$; - Figs. 4I, J, $\mathbf{K}$ ). These observations matched the inflammation laterality of the average X-ray images (-Figs. 4L, $\mathbf{M}, \mathbf{N}$ ).

\section{Discussion}

Sinusitis is defined as the acute or chronic inflammation of the sinuses. Acute inflammation of the sinuses was investigated in the present study because it is associated with fever. However, recent research indicated that thermography is also effective for the diagnosis of chronic sinusitis. ${ }^{5}$ The heat distribution in the paranasal sinus may depend upon its pneumatization; that is, mucosa and/or fluid have higher temperatures than air in the paranasal sinus.

The present statistical study was performed on patients with maxillary sinusitis. The application of thermography on the frontal sinus seemed more effective because the location of the frontal sinus is shallower than that of the maxillary sinus. Conversely, variations in the shape of the frontal sinus made the standardization process difficult. Particularly among female subjects, sometimes, the view of the frontal head region is obstructed during thermography due to the fact that it is covered with hair. Consequently, we focused on analyzing the maxillary sinus.

Although inflammation of the paranasal sinus increases the facial temperature, a reference point is needed for visualization. The terminal of the right side of the face was regarded as a reference point, and it was visualized at the beginning (-Fig. 2A). Although inflammation was observed in the left maxillary sinus on X-ray imaging ( - Fig. 2B), both sides showed a unique pattern on thermography ( - Fig. 2A). In fact, a slight shadow is observed in the right maxillary sinus on X-ray imaging. Therefore, thermography may be more sensitive than X-ray imaging for the detection of inflammation.

A higher temperature was observed in the frontal head in the subjects without sinusitis (-Fig. 3). Because scalp temperature reflects cerebral blood flow, ${ }^{8}$ the upper frontal head temperature was not considered in the present study.

In the original thermography, although the shape of the paranasal sinus was visualized, there was no statistical significance. We concluded that the range of average facial temperatures varies, and that the temperature increases caused by sinusitis were small; therefore, the difference in heat distribution between subjects with and without sinusitis was unclear. However, the difference was significant when the original digital data of thermography were processed by reducing the influence of the average facial temperatures using a relative SST image. Regions with increasing temperatures were located relative to lateral sides ( - Fig. 4), which possibly explains the location of inflammation mucosa in the maxillary sinus.

Because the borderline between the face and the background was unclear in the average image, contamination of the infrared ray background seemed to induce a significant 


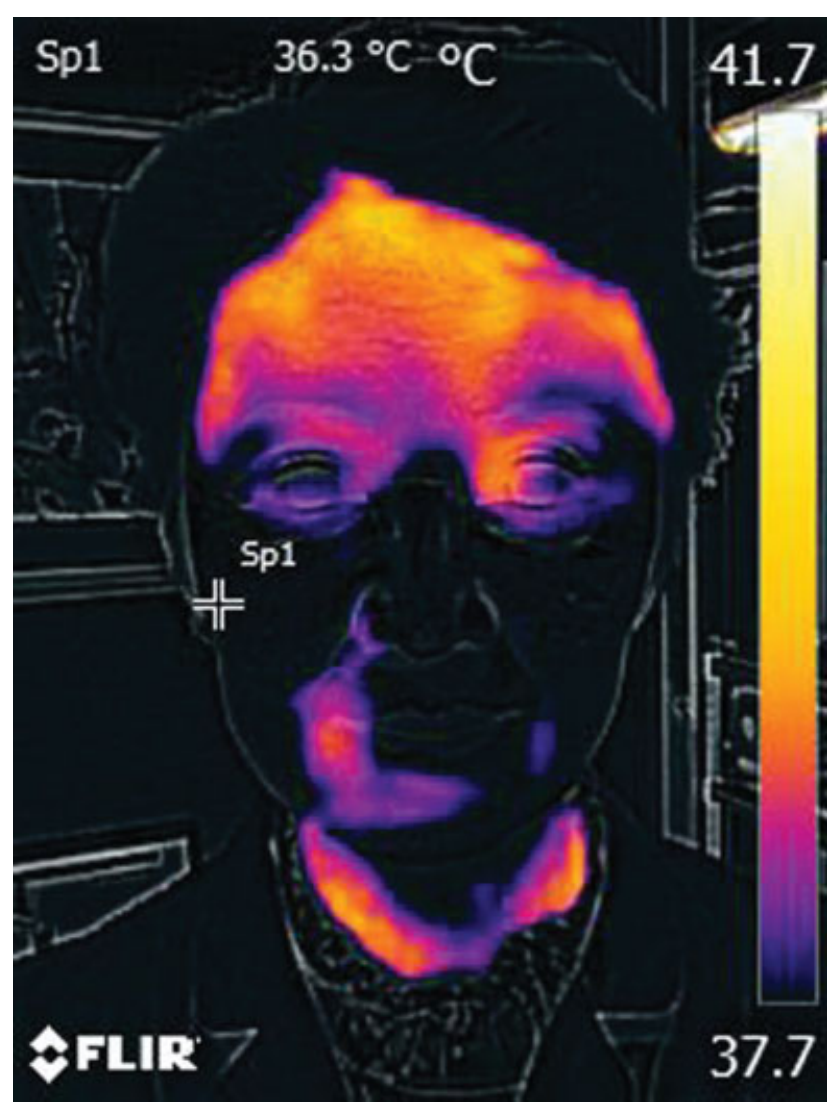

Fig. 3 Pattern of heat distribution on a typical control subject. The threshold level of temperature is set at $37.7^{\circ} \mathrm{C}$. The pattern of heat distribution is not similar to the shape of the paranasal sinus. The temperature $\left({ }^{\circ} \mathrm{C}\right)$ range is indicated as a bar. The subject and the data are the same as those in - Figure $\mathbf{1}$.

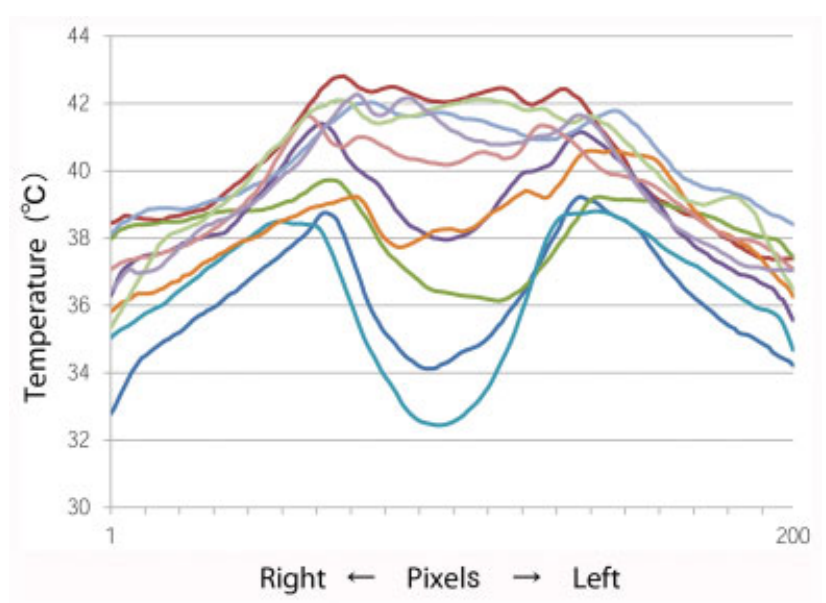

Fig. 5 Horizontal heat distribution in 10 control subjects. The horizontal heat distributions of the maxillary region ( $\mathbf{- F i g . ~} \mathbf{3 A}$ ) of 10 control subjects are plotted as 10 traces. Each pixel value of SST image is averaged from top to bottom to create a horizontal heat distribution.

false positive in the borderline between the face and the background (-Fig. 4l, left).

Heat distribution on the face of healthy subjects is symmetrical, but varies over time. ${ }^{9}$ Facial temperatures are easily influenced by the environment. Gender and racial origin also influence the absolute value of facial temperatures. ${ }^{5,9}$ All subjects were Japanese; therefore, there were no racial differences among the subjects in the present study. We did not investigate gender differences because the number of subjects was insufficient.

Christensen et $\mathrm{al}^{9}$ reported that bilateral regions beside the nose indicate fever in female subjects. If gender influence

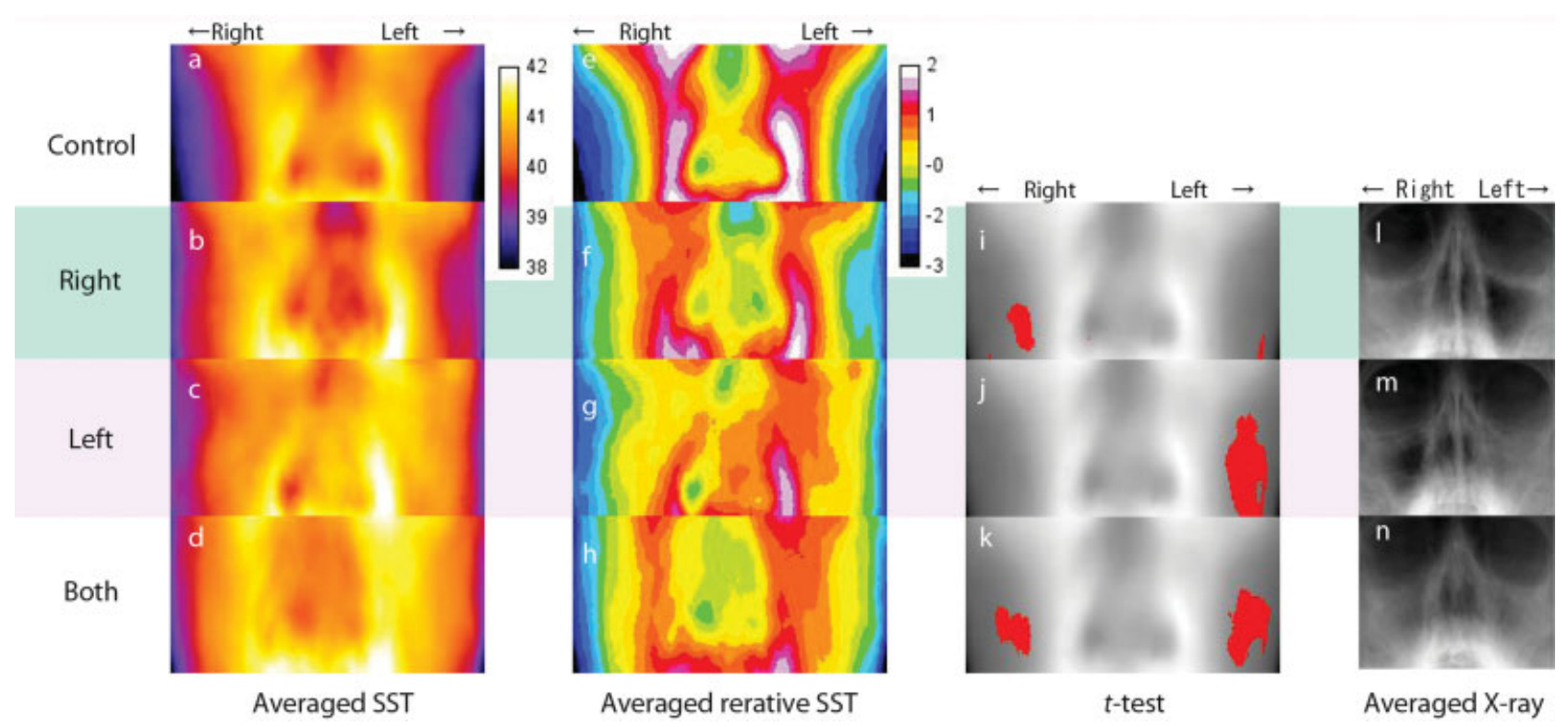

Fig. 4 Average heat distribution and statistical analysis of the maxillary region. Average SST image of the control subjects (A, $n=10)$ and patients with sinusitis (B, right side, $n=6)(C$, left side, $n=6)(D$, bilateral, $n=6)$. The temperature $\left({ }^{\circ} \mathrm{C}\right)$ range is indicated as a bar. Average relative SST image of the control subjects $(E, n=10)$ and patients with sinusitis $(F$, right side, $n=6)(G$, left side, $n=6)(H$, bilateral, $n=6)$. The temperature $\left({ }^{\circ} \mathrm{C}\right)$ range is indicated as a bar. The difference in relative SST images between the control $(\mathrm{E})$ and sinusitis groups $(\mathbf{I}, \mathbf{J}, \mathbf{K})$ were statistically analyzed, and the significant fever regions are indicated as "red" ( $t$ test, $p<0.05$ ). Maxillary images are only used to indicate the location. The average $\mathrm{X}$-ray images of these groups are presented $(\mathrm{L}, \mathrm{M}, \mathrm{N})$. 
is significant, bilaterally-significant fever regions may be observed in the face independent of any influence of sinusitis. In the present study, only the left maxillary temperature was significantly higher in one male and in five female patients with left sinusitis. Despite the higher number of females, bilateral fever spots were not observed. Therefore, even though the ratio of male to female differed between the groups in our study, the influence of gender seemed to be small.

Facial temperature is influenced by odontalgia and dental prosthesis. ${ }^{6}$ There is a significant difference in facial temperature between subjects with and without odontalgia. ${ }^{3}$

In our study, only 1 out of 20 patients had OMS with odontalgia. Although the other 19 patients did not have odontalgia, the heat distribution in the patients with sinusitis was significantly different compared with that of the control subjects. Because averages and $t$ tests were used for the data analysis, the heat effect from sinusitis seemed to be clear, even though most patients did not have odontalgia.

Haddad et $\mathrm{al}^{6}$ reported the importance of the environment in the thermographic examination room. They studied heat distribution on the face, following the practical guide to neuromusculoskeletal thermography. ${ }^{10}$ In this guide, the conditions of the examination room, such as temperature, humidity, speed of incident air, window size, and use of cold light, are regulated. Kusumi et $\mathrm{al}^{2}$ reported that the temperature of the nose was influenced by room temperature. Conversely, Christensen et $\mathrm{al}^{9}$ reported no significant relationship between facial temperatures and room temperature. Because our experiments were performed during the winter, the subjects waited in a warm room with air conditioning. The waiting room temperature was not homogenous. We believe that the preexamination environment may have influenced the thermography results. Although reports on the influence of room temperature are contradictory, it is generally believed that the environment in the roomis important, and that its influence may be suppressed in the relative SST images.

The present study found no significant correlation between body temperature and average facial temperatures, which is consistent with the results of Christensen et al. ${ }^{9}$ It is thought that body temperature is not a useful parameter for the visualization of sinus inflammation via thermography. For the clinical diagnosis, it may be desirable to perform examinations in a simple way without using the special purpose examination room. It seemed that digital image processing reduced the influence of examination room conditions. The relative SST image method decreased the errors generated by room conditions, and may enable the diagnosis of sinusitis.

Maxillary sinusitis increases facial temperature. Therefore, inflammation-dependent heat distribution was statistically visualized, and thermography was shown to correlate with X-ray imaging in sinusitis. Although this method requires a personal computer (PC) for image processing, it can be implemented without a PC if a dedicated iOS application is developed. Thus, if this method is evolved, it can be used by a general physician at a patient's home. Also, since no radiation is used, not only a physician but also a nurse can use it. Thermography is a promising technology for the visualization of sinusitis.

\section{Conclusion}

In the present study, differences in heat distribution in subjects with and without sinusitis were investigated using thermography. Significant fever regions were observed through relative thermography data. Therefore, thermography provides an additional method to visualize sinusitis.

\section{Funding}

The authors received no financial support for the research, authorship, and/or publication of the present article.

\section{Conflict of Interests}

The authors have none to declare.

\section{References}

1 Sitnikov VP, Egorova TA. Thermographic and rhinorheographic parallels in clinical diagnosis of inflammatory diseases of paranasal sinuses. Vestn Otorinolaringol 1989;4(04):53-56 In Russian

2 Kusumi T, Ishii K, Kusumi M, et al. A study of facial skin temperature by thermographic examination in relation to environmental temperature. J Showa Med Assoc 1989;49:480-487 In Japanese

3 Sergeev SV, Grigor'kina ES, Smogunov VV, Kuz'min AV, Volkova NA. The combined application of thermography and local thermometry for diagnostics, prognostication, modeling, and evaluation of the effectiveness of the treatment of acute sinusitis. Vestn Otorinolaringol 2014;5(05):52-54 In Russian

4 Niedzielska I, Pawelec S, Puszczewicz Z. The employment of thermographic examinations in the diagnostics of diseases of the paranasal sinuses. Dentomaxillofac Radiol 2017;46(06): 20160367. DOI: 10.1259/dmfr.20160367

5 Kalaiarasi R, Vijayakumar C, Archana R, et al. Role of Thermography in the Diagnosis of Chronic Sinusitis. Cureus 2018;10(03): e2298. DOI: 10.7759/cureus.2298

6 Haddad DS, Brioschi ML, Baladi MG, Arita ES. A new evaluation of heat distribution on facial skin surface by infrared thermography. Dentomaxillofac Radiol 2016;45(04):20150264. DOI: 10.1259/ dmfr.20150264

7 Fokkens WJ, Lund VJ, Mullol J, et al. European Position Paper on Rhinosinusitis and Nasal Polyps 2012. Rhinol Suppl 2012;23:3, 1298

8 Shevelev IA. Functional imaging of the brain by infrared radiation (thermoencephaloscopy). Prog Neurobiol 1998;56(03):269-305

9 Christensen J, Vaeth M, Wenzel A. Thermographic imaging of facial skin-gender differences and temperature changes over time in healthy subjects. Dentomaxillofac Radiol 2012;41(08): 662-667

10 Schwaltz RG. Guidelines for neuromusculoskeletal thermography. Thermol Int 2006;16:5-9 